again" to "daily" over the past 6 months. For the latter purpose all participants were identified with their work-unit (471 work units, number of employees ranging between 1 and 161). The work-units were grouped according to the proportion of employees. who had witnessed workplace bullying within their work units. New cases of depression were diagnosed at the end of two-year follow-up periods using Schedules for Clinical Assessment in Neuropsychiatry (SCAN) interviews and the Major Depression Inventory questionnaire.

Results During the follow-up period, we identified 177 new cases of depression. The odds ratio for newly-onset depression among participants reporting bullying occasionally was 1.62 [95\% CI 0.95-2.77] and among those reporting bullying often it was 5.73 [95\% CI 2.37-13.90]. The risk of newly-onset depression by percentage of employees witnessing bullying in work-units was for 1-20\%: 0.83 [95\% CI 0.48-1.43], 2130\%: 0.87 [95\% CI $0.49-1.55$ ], and >30\%: 1.08 [95\% CI 0.61-1.90].

Conclusions Self-reported frequent bullying predicts development of depression but a work environment defined by witnesses of bullying does not. These findings have implications for the understanding of workplace bullying and options for preventive actions.

\section{SEX DIFFERENCES IN MUSCULAR LOAD AMONG HOUSE PAINTERS PERFORMING IDENTICAL WORK TASKS}

${ }^{1} \mathrm{~T}$ H H Heilskov-Hansen, ${ }^{2}$ Meyland, ${ }^{3}$ Alkjær, ${ }^{3}$ Koblauch, ${ }^{2}$ Mikkelsen, ${ }^{4}$ Wulff Svendsen, ${ }^{2}$ Frølund Thomsen, ${ }^{5}$ Hansson, ${ }^{3}$ Bruun Simonsen. 'Bispebjerg University Hospital, Copenhagen NV, Denmark; '2Department of Occupational and Environmental Medicine, Bispebjerg University Hos, Copenhagen, Denmark; ${ }^{3}$ Department of Neuroscience and Pharmacology, University of Copenhagen, Copenhagen, Denmark; ${ }^{4}$ Danish Ramazzini Centre, University Department of Occupational Medicine, Herning, Herning, Denmark; ${ }^{5}$ Occupational and Environmental Medicine, Lund University, and University and Reg, Lund, Sweden

\subsection{6/oemed-2013-101717.293}

Objective The aim of the present study was to estimate possible differences in upper body muscular load between male and female house painters performing identical work tasks. Sexrelated differences in muscular activity may help explain why female house painters, and females in general, have more musculoskeletal complaints and disorders than men do.

Methods In a laboratory-setting, 16 male and 16 female house painters performed nine standardised work tasks common to house painters. Unilateral EMG recordings were obtained from the supraspinatus muscle by intramuscular electrodes and from the trapezius-, extensor- and flexor carpi radialis muscles by surface electrodes. Maximum voluntary contractions were performed, and both relative muscular load in $\% \mathrm{EMG}_{\max }$ as well as exerted force in Newton were assessed. APDF curves were obtained for each subject, and load distributions were characterised by the $10^{\text {th }}, 50^{\text {th }}$ and $90^{\text {th }}$ percentiles; sex differences were tested using a mixed model approach.

Results Women were exposed to a significantly $(\mathrm{P}=0.05)$ higher relative muscular load than men in the supraspinatus and forearm muscles in all tasks. Men exerted significantly $(\mathrm{P}=$ $0.05)$ more absolute force in the trapezius muscle at the $50^{\text {th }}$ percentile in all tasks, and in a single task also at the $10^{\text {th }}$ percentile. The differences between men and women were independent of tasks.

Conclusion Female house painters had a higher relative muscular load than their male colleagues, even though the men exerted more absolute force compared to the women. The effects of a higher relative muscular load, accumulated over years of work, may in part explain why musculoskeletal complaints and disorders in the upper body occurs more frequently among female than male house painters.

\section{DOES DEPRESSION SEVERITY PREDICT DIFFERENCES BETWEEN INDIVIDUAL AND WORK-UNIT AVERAGED MEASURES OF THE PSYCHOSOCIAL WORK ENVIRONMENT?}

${ }^{1} \mathrm{M}$ W Willert, ${ }^{2}$ Persson, ${ }^{2}$ Hansen, ${ }^{3}$ Mors, ${ }^{4}$ Thomsen, ${ }^{4}$ Bonde, ${ }^{5}$ Kolstad. ${ }^{1}$ Aarhus University Hospital, Aarhus, Denmark; ${ }^{2}$ The National Research Centre for the Working Environment, Copenhagen, Denmark; 'Aarhus University Hospital, Risskov, Aarhus, Denmark; ${ }^{4}$ Department of Occupational and Environmental Medicine, Bispebjerg Hospital, Copenhagen, Denmark; ${ }^{5}$ Department of Occupational Medicine, Danish Ramazzini Centre, Aarhus University Hospital, Aarhus, Denmark

\subsection{6/oemed-2013-101717.294}

Objectives It is debated if results from epidemiological studies on psychosocial factors at work and risk of depression are affected by the common rater problem, thus inflating associations by introducing reporting bias. Because depression is associated with cognitive distortions it is expected that severity of depression affects the level of reported exposures. The present study aims to investigate the possible dose-effect relationship between diagnosed severity of depression and differences between individual and work-unit averaged measures of the psychosocial work environment in a large epidemiological study. Methods In 20074291 Danish public employees within 378 different work units were enrolled in the study. Mean levels of psychological demands were computed for each work unit. Screening for depressive symptoms (SCL-DEP6) 329 persons scoring above cut-off were invited to a diagnostic interview (SCAN), resulting in 40 cases with mild, 43 with moderate, and 17 with severe depression, and 229 cases with no diagnosis of depression. Differences were analysed with ANOVA statistics.

Results For quantitative demands the mean score difference was 0.08 points for participants with mild depression compared to their work-unit average, 0.28 points for participants with moderate depression and 0.36 points for participants with severe depression $(\mathrm{p}=0.40)$.

Conclusions The data indicate a dose-effect pattern between severity of depression and divergence from work-unit averages. However, the results are not statistically significant and our initial hypothesis is not supported. Future perspectives involve including two follow-up waves to gather more cases and provide additional statistical power, and also allow adjustment for relevant confounders and changes in reporting over time. These results have implications regarding the need for exposure measures that are independent of reports from participants diagnosed with depression, but may also be explored as indicators of social marginalisation as a mechanism relevant to the development or maintenance of depression.

\section{ERGONOMIC EXPOSURE ASSESSED BY PRODUCTION STATISTICS}

${ }^{1} \mathrm{C}$ B Brauer, 'Bern, ${ }^{2}$ Alkjaer, 'Bonde, ${ }^{3}$ Helweg-Larsen, ${ }^{2}$ Koblauch, ${ }^{3}$ Moller, ${ }^{2}$ Simonsen, ${ }^{1}$ Thomsen, ${ }^{3}$ Thygesen, ${ }^{1}$ Mikkelsen. ${ }^{1}$ Department of Occupational and Environmental Medicine, Bispebjerg University Hospital, Copenhagen NV, Denmark; '2Department of Neuroscience and Pharmacology, University of Copenhagen, Copenhagen, Denmark; 
${ }^{3}$ National Institute of Public Health, University of Southern Denmark, Copenhagen, Denmark

\subsection{6/oemed-2013-101717.295}

Objectives To assess ergonomic exposures in a historical cohort study on musculoskeletal disorders among baggage handlers using production statistics from the airport and the involved handling company.

In historical cohort studies information on exposures is typically based on self-reports or assumptions of previous exposures. This may lead to misclassification of cumulative exposures. We analysed production data from the participating companies to improve the exposure assessments of the individual workers.

Methods Data from 1990-2012 regarding the number of flight operations was provided by Copenhagen Airport. The handling company provided data from 1998-2012 on total baggage weight, number of operations and number of baggage handlers on duty. Additionally, information existed back to 2002 on type of aircrafts, percentage of wide-bodied aircrafts (where some of the baggage was loaded in containers) and weight of manually handled baggage. It was possible to receive information about the dimension of the baggage compartments, which is decisive for the postures adopted in the hold (standing, stooping, squatting, kneeling and sitting). Information also existed on the time of introduction of technical lifting gear.

Results Although the number of passengers almost doubled during the past 20 years, the number of flight operations decreased as the number of passengers per flight increased. However, the weight of manually handled luggage out on the ramp decreased, probably because the number of wide-bodied aircrafts increased. Seasonal and daily variations existed. Because of the detailed production statistics it was possible to create a job exposure matrix by job tasks and calendar periods. This information will be combined with individual information on job tasks back in time.

Conclusion Although production statistics cannot provide information on individual factors such as the worker's lifting technique and his use of lifting gear, production statistics may be a valuable tool in exposure assessments in epidemiological studies.

\section{INFLUENCE FROM NON-OCCUPATIONAL FACTORS ON SELF-REPORTED OCCUPATIONAL LIFTING AMONG PREGNANT WOMEN: FINDINGS FROM THE DANISH NATIONAL BIRTH COHORT}

${ }^{1} \mathrm{P}$ F Frost, ${ }^{2}$ Svendsen, ${ }^{3}$ Mocevic, ${ }^{3}$ Jørgensen, ${ }^{4}$ Nybo Andersen, ${ }^{3}$ Bonde. ${ }^{1}$ Aarhus University Hospital, Aarhus C, Denmark; 'Herning Regional Hospital, Herning, Denmark; ${ }^{3}$ Bispebjerg University Hospital, Copenhagen, Denmark; ${ }^{4}$ University of Copenhagen, Copenhagen, Denmark

\subsection{6/oemed-2013-101717.296}

Objective To establish exposure estimates based on prospective interviews in a cohort of pregnant women using a job and industry group-based approach, and to evaluate whether non-occupational factors should be considered when a group-based strategy is chosen based on self-report.

Methods Within the Danish National Birth Cohort, a job exposure matrix (JEM) based on information from participants still pregnant at date of interview had been constructed. Job titles were coded using the Danish version of the International Standard Classification of Occupations (DISCO-88) and the Danish
Industrial Classification of All Economic Activities of employment industry was obtained from Statistics Denmark. We calculated the mean number of kilograms lifted per day within groups defined firstly by identical four digits DISCO- 88 codes and employment industry, secondly by identical four, three or two digits DISCO-88 codes, depending on a compulsory group size of $\geq 10$ participants. Level of self reported occupational lifting in relation to JEM-estimate and non-occupational factors including gestational age at interview, mother's age, parity, smoking and body mass index (bmi) was analysed with restriction to participants with identical job and industry codes. We used multivariate regression with bootstrap to obtain regression coefficients and 95\% confidence intervals.

Results A total of 41,405 women were included in the analyses. Gestational age $>97$ days at interview and increasing mother's age were associated with reporting of lower levels of occupational lifting, whereas bmi $\geq 30 \mathrm{~kg} / \mathrm{m}^{2}$, smoking while pregnant, and being a mother was associated with reporting of higher levels. Differences ranged from $-15 \mathrm{~kg}$ to $35 \mathrm{~kg}$.

Conclusion We constructed a JEM based on self-report to obtain group-based exposure estimates, striving to minimise information bias and attenuation in exposure response analyses. Non-occupational factors influence reporting of occupational lifting among pregnant women. This should be considered when a group-based strategy is used for exposure assessment based on self-report.

\section{GROUP-BASED EXPOSURE MEASUREMENT STRATEGIES AND THEIR EFFECTS ON TRUNK ROTATION AND LOW- BACK PAIN EXPOSURE-OUTCOME ASSOCIATIONS}

${ }^{1} \mathrm{P}$ Coenen, ${ }^{2}$ Mathiassen, ${ }^{3}$ Kingma, ${ }^{4}$ Boot, ${ }^{5}$ Bongers, ${ }^{3}$ van Van Dieen. ${ }^{1}$ Vrije Universiteit, Amsterdam, The Netherlands; '2University of Gavle, Gavle, Sweden; ${ }^{3} \mathrm{VU}$ University, Amsterdam, Nederland; ${ }^{4}$ VU Medical Center, Amsterdam, Nederland; ${ }^{5}$ TNO Healthy Living, Hoofddorp, Nederland

\subsection{6/oemed-2013-101717.297}

Objectives In epidemiological studies of occupational exposures (e.g. lifting) and low-back pain (LBP), group-based exposure measurement strategies are common. Workers are classified into exposure groups; exposure is measured only in a selection of workers in each group, and their mean exposure is assigned to all workers in the group. Exposure-outcome relationships are then determined by regression, relating exposure estimates with individual LBP data from all subjects. The objective of this study was to assess the effect of different group-based measurement strategies on exposure-outcome associations.

Methods 1122 workers, classified into 19 groups on the basis of job-related exposure, participated in this study. In each group, videos were collected from $\sim 25 \%$ of the workers (in total, 370 workers), and percentage of the work day spent in trunk rotation was estimated by observation of the videos. This estimate of trunk rotation was significantly associated with self-reported LBP during three years of follow-up (OR:1.43 (1.06-1.93)).

Using a bootstrap simulation, workers per group $(n=10,20$, $30,40)$ and percentage of observed workers $(\mathrm{k}=10,20,30,40$, $50 \%$ ) were varied. For each combination, (nk) workers were selected with replacement in each job group among those observed, and n (100-k) workers among those not observed. The mean exposure of the observed workers was assigned to all group members which was related to individual LBP data. ORs and accompanying p-level was estimated using logistic-regression.

Results A group-based measurement protocol led to significant $(\mathrm{p}<0.05)$ ORs when the total number of workers was larger 